\title{
ON ASYMPTOTIC BEHAVIOR OF POSITIVE SOLUTIONS TO SOME QUASILINEAR INEQUALITIES ON MODEL RIEMANNIAN MANIFOLDS
}

\author{
A.G. LOSEV, E.A. MAZEPA
}

\begin{abstract}
In the paper we study asymptotic behavior of positive solutions to some quasilinear elliptic inequalities on spherically symmetric noncompact (model) Riemannian manifolds. In particular, we find conditions under which Liouville type theorems on absence of nontrivial solutions hold true, as well as the conditions of existence and cardinality of the set of positive solutions of the studied inequalities on the Riemannian manifolds. The results generalize similar results obtained previously by Y. Naito and H. Usami for the Euclidean space $\mathbb{R}^{n}$.
\end{abstract}

Keywords: quasilinear elliptic inequality, Liouville type theorem, model Riemannian manifolds.

\section{INTRODUCTION AND MAIN RESULTS}

This work is devoted to studying the asymptotic behavior of solutions to the inequality

$$
L u \equiv \operatorname{div}(A(|\nabla u|) \nabla u) \geq f(u)
$$

on model Riemannian manifolds. In particular, we find the conditions for validity of Liouville type theorems on the absence of nontrivial solutions to inequality (1).

As one of the origins of these problems, one traditionally mentions the classification theory for two-dimensional Riemannian surfaces. The uniformization theorem implies that each simplyconnected Riemannian manifold is conformally equivalent to one of the following model surfaces,

1) the sphere (elliptic surface);

2) the complex plane (parabolic surface);

3) the unit disk or, that is the same, the hyperbolic plane with its complex analytic structure (hyperbolic surface).

The feature of two-dimensional parabolic (hyperbolic) surfaces is the validity (or failure) of Liouville theorem saying that each positive superharmonic function on this surface is identically constant. Exactly this property served as a basis for the extension of the parabolicity notion for arbitrary Riemannian manifolds. Namely, manifolds on which any lower bounded superharmonic function is constant are called parabolic manifolds.

During last years there was published a series of papers devoted to the questions on validity of Liouville type theorems for various classes of solutions and subsolutions of linear equations on non-compact Riemannian manifolds. The general overview on modern studies on this subject can be obtained, for instance, from the survey by A.A. Grigor'yan, see [1].

A.G. Losev, E.A. Mazepa, On asymptotic Behavior of Positive Solutions of some Quasilinear INEQUALITIES ON MODEL RIEMANNIAN MANIFOLDS.

(C) Losev A.G., Mazepa E.A. 2013.

The work is supported by RFBR (grant no. 10-01-97004-r_povolzhe_a).

Submitted November 29, 2011. 
In particular, the exact conditions of validity of Liouville type theorems for solutions of some equations and inequalities on spherically-symmetric or model Riemannian manifolds were obtained in works [2]-[4]. Let us describe these manifolds in more details.

We fix the origin $O \in \mathbb{R}^{n}$ and a smooth function $q$ on the interval $[0, \infty)$ such that $q(0)=0$ and $q^{\prime}(0)=1$. We define a model Riemannian manifold $M_{q}$ as follows,

1) the set of the points of $M_{q}$ is $\mathbf{R}^{n}$;

2) in polar coordinates $(r, \theta)$ (where $r \in(0, \infty)$ and $\theta \in S^{n-1}$ ), the Riemann metrics on $M_{q} \backslash\{O\}$ is defined as

$$
d s^{2}=d r^{2}+q^{2}(r) d \theta^{2}
$$

where $d \theta$ is the standard Riemann metrics on the sphere $S^{n-1}$;

3 ) the Riemann metrics at the point $O$ is a smooth continuation of metrics (2).

In what follows the function $A$ in inequality (1) is assumed to satisfy the following conditions,

$$
\left\{\begin{array}{l}
A \in C(0, \infty), \quad A(p)>0 \quad \text { as } p>0 \\
p A(|p|) \in C(\mathbb{R}) \cap C^{1}(0, \infty), \\
(p A(p))^{\prime}>0 \text { for } p>0
\end{array}\right.
$$

and the function $f \not \equiv 0$ is so that

$$
f \in C(0, \infty), \quad f(u) \geq 0 \text { as } u \geq 0 \text { and } f(0)=0 .
$$

We shall make use of the following assumption for the function $f$,

(F) $\left\{\begin{array}{l}\text { there exists a non-decaying function } g \in C(0, \infty) \text { such that } \\ 0<g(u) \leqslant f(u) \text { as } u>0 \text { and } g(0)=0 .\end{array}\right.$

The equations of this kind were considered by many authors in Euclidean spaces (see, for instance, [5]-[8]). The functions $A(p)$ most frequently appearing in studies are of the following types,

$$
\begin{gathered}
A(p)=p^{m-2}, \quad m>1 ; \\
A(p)=\left(1+p^{2}\right)^{-\frac{1}{2}}
\end{gathered}
$$

or, in a more general form,

$$
A(p)=\left(1+p^{2}\right)^{-\alpha}, \quad \alpha \leqslant \frac{1}{2} .
$$

According to that, the operator $L$ in inequality (1) is called $m$-Laplacian under the choice of the function $A$ as (3), the mean curvature operator in case (4), and generalized mean curvature operator in case (5) as $0<\alpha<1 / 2$.

By an entire solution to inequality (1) on Riemannian manifold $M$ we shall call a function $u \in C^{1}(M)$ such that $A(|\nabla u|) \nabla u \in C^{1}(M)$ and obeying inequality (1) at each point $x \in M$.

In the simplest case $A(p) \equiv 1$, the problem of existence of entire solutions to inequality (1) in $\mathbb{R}^{n}$ was studied in the series of paper. In particular, if $f$ is a non-decaying function, Keller J. and Osserman R. (see [6] and [7]) showed that the inequality $\Delta u \geq f(u)$ had positive entire solutions if and only if

$$
\int^{\infty}\left(\int^{s} f(t) d t\right)^{-\frac{1}{2}} d s=\infty .
$$

In work [5] Naito Y. and Usami H. generalized their result for inequality (1) and obtained the criterion of existence of nontrivial nonnegative solutions to inequality (1) in $\mathbb{R}^{n}$. The aim of the present work is to obtain similar results on a class of model Riemannian manifolds. At that, it is natural to consider the operator $L$ in inequality (1) in metrics (2) of the manifold $M$. 
First we consider the case $\lim _{p \rightarrow \infty} p A(p)<\infty$. We introduce the notation

$$
I(r)=\frac{1}{q^{n-1}(r)} \int_{0}^{r} q^{n-1}(s) d s .
$$

We note that the conditions for the metrics of the model Riemannian manifold immediately imply $I(0)=\lim _{r \rightarrow+0} I(r)=0$.

Theorem 1. Suppose $\lim _{p \rightarrow \infty} p A(p)<\infty$ and the manifold $M_{q}$ is so that $\limsup _{r \rightarrow \infty} I(r)=\infty$. If condition $(\mathrm{F})$ is satisfied, there exists no entire positive solutions to inequality (1) on $M_{q}$.

Then we consider the case $\lim _{p \rightarrow \infty} p A(p)=\infty$. We define a continuous function $\Psi:[0, \infty) \rightarrow$ $[0, \infty)$ as

$$
\Psi(p)=p^{2} A(p)-\int_{0}^{p} t A(t) d t, \quad p \geq 0 .
$$

It is easy to show that $\Psi$ is strictly increasing and $\Psi(0)=0$. We also mention that as $p \geq 1$, the identity

$$
\Psi(p)+\int_{0}^{1} t A(t) d t=p^{2} A(p)-\int_{1}^{p} t A(t) d t \geq p A(p)
$$

holds true that yields $\lim _{p \rightarrow \infty} \Psi(p)=\infty$.

Thus, the inverse to $\Psi$ function $\Phi$ is defined on $[0, \infty)$. It is clear that $\Phi$ is a strictly increasing function and $\lim _{p \rightarrow \infty} \Phi(p)=\infty$.

Theorem 2. Suppose $\lim _{p \rightarrow \infty} p A(p)=\infty$ and the manifold $M_{q}$ is such that $I^{\prime}(r) \geq k>0$. If condition $(\mathrm{F})$ is satisfied and moreover

$$
\int^{\infty}\left(\Phi\left(k \int^{s} g(t) d t\right)\right)^{-1} d s<\infty
$$

there exists no entire positive solutions to inequality (1) on $M_{q}$.

Theorem 3. Suppose $\lim _{p \rightarrow \infty} p A(p)=\infty$ and the manifold $M_{q}$ is such that $q^{\prime}(r) \geq 0$. If

$$
\int^{\infty}\left(\Phi\left(\int^{s} f(t) d t\right)\right)^{-1} d s=\infty
$$

inequality (1) has continuum of positive entire solutions.

\section{RADIAL SOLUTIONS}

In the beginning of the present section we formulate an analogue of comparison principle obtained in work [5], on which the further arguments are based.

Lemma 1 ([5]). Suppose $\Omega \subset M$ is a bounded domain with a smooth boundary $\partial \Omega$, $u$ is a nonnegative solution to (1) in $\Omega$, and $v \in C(\bar{\Omega}) \bigcap C^{1}(\Omega)$ is a positive function such that $A(|\nabla v|) \nabla v \in C^{1}(\Omega), L v \leqslant g(v)$ in $\Omega$, and $u \leqslant v$ on the boundary $\partial \Omega$. If condition $(\mathrm{F})$ is satisfied, then $u \leqslant v$ in $\Omega$. 
The key ingredient of the statements being proven is the study of radially symmetric solutions $v(r)$ of the considered inequalities. It is easy to show that on a model manifold $M_{q}$

$$
L v(r) \equiv \operatorname{div}(A(|\nabla v(r)|) \nabla v(r))=q^{1-n}(r)\left(q^{n-1}(r) A\left(\left|v^{\prime}(r)\right|\right) v^{\prime}(r)\right)^{\prime} .
$$

Let us consider the following ordinary differential equation

$$
\left(q^{n-1}(r) A\left(\left|v^{\prime}(r)\right|\right) v^{\prime}(r)\right)^{\prime}=q^{n-1}(r) g(v(r)), \quad r \geq 0,
$$

where $g$ is continuous positive non-decaying on $(0, \infty)$ function in condition $(\mathrm{F})$.

Let $v(r)$ be the solution to equation $\sqrt{80}$ with initial data $v(0)>0$ and $v^{\prime}(0)=0$. We observe that if $v(r)$ is defined for $0 \leqslant r<R \leqslant \infty$, then $v^{\prime}(r)>0$ for $0<r<R$. Indeed, integrating identity (8) over segment [0, $r], r<R$, we obtain

$$
A\left(\left|v^{\prime}(r)\right|\right) v^{\prime}(r)=q^{1-n}(r) \int_{0}^{r} q^{n-1}(s) g(v(s)) d s, \quad 0<r<R .
$$

Therefore, $A\left(\left|v^{\prime}(r)\right|\right) v^{\prime}(r)>0$ for $0<r<R$ that implies $v^{\prime}(r)>0$ as $0<r<R$.

Lemma 2. Suppose condition (F) holds true. If inequality (1) has a positive entire solution $u(r, \theta)>0$ on $M_{q}$, on $[0 ; \infty)$ there exists a positive solution $v(r)$ to equation (8) subject to conditions $v(0)>0$ and $v^{\prime}(0)=0$.

Proof. Suppose the opposite, i.e., there exists no solution $v(r)$ to equation (8) described in the statement of the lemma, but at the same time there exists a positive entire solution $u(r, \theta)$ of inequality (1). The positivity of solution to inequality in particular implies that $u(O)>0$. Let $a \in(0, u(O))$, and $[0 ; R)$ be the maximal segment of the existence for the solution $v$ to equation (8) subject to the conditions $v(0)=a$ and $v^{\prime}(0)=0$. By the assumption $R<\infty$. We have shown above that $v^{\prime}(r)>0$ as $0<r<R$. Then we have either $v(r) \rightarrow \infty$ as $r \rightarrow R$ or $v^{\prime}(r) \rightarrow \infty$ as $r \rightarrow R$.

In the case $v(r) \rightarrow \infty$ as $r \rightarrow R$ we choose $R_{1} \in(0, R)$ so that

$$
v\left(R_{1}\right)>\max _{\Omega} u(r, \theta),
$$

where $\Omega \equiv B_{R_{1}}=\left\{(r, \theta): r \in\left[0, R_{1}\right]\right\}$. Then $L v=g(v)$ in $\Omega$ and $v \geq u$ on $\partial \Omega$. Therefore, by Lemma $1, u \leqslant v$ in $\Omega$ that contradicts the condition $v(0)=a<u(O)$.

Consider the case $v^{\prime}(r) \rightarrow \infty$ as $r \rightarrow R$. If there exists $R_{1} \in(0 ; R)$ such that inequality (10) is satisfied, we obtain a contradiction similar to one obtained above. Let $v(r) \leqslant \max _{\theta \in S^{n-1}} u(r, \theta)$ for all $0<r<R$. We choose $R_{1} \in(0 ; R)$ so that

$$
v^{\prime}\left(R_{1}\right)>\max _{\Omega}\left\{\frac{\partial u}{\partial r}(r, \theta)\right\},
$$

where $\Omega \equiv B_{R_{1}}=\left\{(r, \theta): r \in\left[0, R_{1}\right]\right\}$. Denote $\delta=\max _{\theta \in S^{n-1}}\left(u\left(R_{1}, \theta\right)-v\left(R_{1}\right)\right)>0$ and let $w(r)=v(r)+\delta$. Then $w\left(R_{1}\right) \geq u\left(R_{1}, \theta\right)$ for all $\theta \in S^{n-1}$ and $w\left(R_{1}\right)=u\left(R_{1}, \theta^{*}\right)$ for some $\theta^{*} \in S^{n-1}$. Then $L w \leqslant g(w)$ in the domain $\Omega, w \geq u$ on the boundary $\partial \Omega$ and by Lemma we get $w \geq u$ in $\Omega$.

Taking into consideration the conditions $w\left(R_{1}\right)=u\left(R_{1}, \theta^{*}\right), w(r) \geq u(r, \theta)$ as $(r, \theta) \in B_{R_{1}}$, we obtain

$$
v^{\prime}\left(R_{1}\right)=w^{\prime}\left(R_{1}\right) \leqslant \frac{\partial u}{\partial r}\left(R_{1}, \theta^{*}\right)
$$

that contradicts (11). The proof is complete. 


\section{PROOFS OF THEOREMS}

Proof of Theorem 1. Suppose the opposite, namely, that inequality (1) has an entire solution $u(r, \theta)>0$. Then Lemma 2 implies the existence of positive solution $v(r)$ to equation (8) subject to initial conditions $v(0)>0$ and $v^{\prime}(0)=0$ on the ray $[0 ; \infty)$.

Since $g(v)$ and $v(r)$ are non-decaying functions, it follows from $(9)$ that

$$
A\left(\left|v^{\prime}(r)\right|\right) v^{\prime}(r) \leqslant g(v(r)) I(r) .
$$

We note that equation (8) can be represented as

$$
\left(A\left(\left|v^{\prime}(r)\right|\right) v^{\prime}(r)\right)^{\prime}+(n-1) \frac{q^{\prime}(r)}{q(r)} A\left(\left|v^{\prime}(r)\right|\right) v^{\prime}(r)=g(v(r)) .
$$

Combining (12) and (13), we obtain

$$
\left(A\left(\left|v^{\prime}\right|\right) v^{\prime}\right)^{\prime} \geq g(v(r))\left[1-(n-1) \frac{q^{\prime}(r)}{q(r)} I(r)\right]=g(v(r)) I^{\prime}(r) .
$$

We recall that $I(0)=0$. Integrating inequality (14) over the segment $[0 ; r]$, we obtain

$$
A\left(\left|v^{\prime}(r)\right|\right) v^{\prime}(r) \geq \int_{0}^{r} g(v(s)) I^{\prime}(s) d s \geq g(v(0)) I(r) .
$$

The conditions for the functions $A$ follow that

$$
g(v(0)) I(r) \leq A\left(\left|v^{\prime}(r)\right|\right) v^{\prime}(r) \leqslant \lim _{p \rightarrow \infty} p A(p)<\infty, \quad r>0 .
$$

Letting $r \rightarrow \infty$ and passing to the upper limit in the left hand side of the inequality, we arrive at the contradiction with the finiteness of the right hand side. The proof is complete.

Proof of Theorem 2. Suppose inequality (1) has a positive entire solution $u(r, \theta)>0$. Then Lemma 2 yields that on $[0, \infty)$ there exists a solution $v(r)$ to equation (8) subject to initial conditions $v(0)>0$ and $v^{\prime}(0)=0$. It follows from (15) and the hypothesis of the theorem that $\lim _{r \rightarrow \infty} v^{\prime}(r)=\infty$ as $r \rightarrow \infty$. Hence, $\lim _{r \rightarrow \infty} v(r)=\infty$. Multiplying inequality (14) by $v^{\prime}>0$ and integrating over the segment $[0 ; r]$, we obtain

$$
\int_{0}^{r}\left(A\left(\left|v^{\prime}\right|\right) v^{\prime}\right)^{\prime} v^{\prime} d s \geq \int_{0}^{r} g(v(s)) I^{\prime}(s) v^{\prime}(s) d s \geq k \int_{0}^{r} g(v(s)) v^{\prime}(s) d s=k \int_{v(0)}^{v(r)} g(t) d t .
$$

On the the hand, applying integration by parts, we get

$$
\int_{0}^{r}\left(A\left(\left|v^{\prime}\right|\right) v^{\prime}\right)^{\prime} v^{\prime} d s=\int_{0}^{r} v^{\prime} d\left(A\left(\left|v^{\prime}\right|\right) v^{\prime}\right)=\left(v^{\prime}(r)\right)^{2} A\left(\left|v^{\prime}(r)\right|\right)-\int_{0}^{v^{\prime}(r)} A(t) t d t=\Psi\left(v^{\prime}(r)\right) .
$$

Therefore,

$$
\Psi\left(v^{\prime}(r)\right) \geq k \int_{v(0)}^{v(r)} g(t) d t
$$

Passing to the inverse function $\Phi$, by the latter inequality we obtain

$$
\left(\Phi\left(k \int_{v(0)}^{v(r)} g(s) d s\right)\right)^{-1} \cdot v^{\prime}(r) \geq 1, \quad r>0 .
$$


Integrating the obtained inequality over the segment $[0 ; r]$, we get

$$
\int_{v(0)}^{v(r)}\left(\Phi\left(k \int_{v(0)}^{s} g(t) d t\right)\right)^{-1} d s \geq r .
$$

Passing in 16 to the limit as $r \rightarrow \infty$, we have

$$
\int_{v(0)}^{\infty}\left(\Phi\left(k \int_{v(0)}^{s} g(t) d t\right)\right)^{-1} d s=\infty
$$

that contradicts to (6). The proof is complete.

Proof of Theorem 3. To prove the theorem, it is sufficient to prove the existence of a positive solution $v(r)$ to the equation

$$
\left(q^{n-1}(r) A\left(\left|v^{\prime}(r)\right|\right) v^{\prime}(r)\right)^{\prime}=q^{n-1}(r) f(v(r))
$$

on the interval $[0 ; \infty)$ subject to the initial conditions $v(0)>0$ and $v^{\prime}(0)=0$, since the function $v(r)$ a radially-symmetric positive entire solution to inequality (1).

We choose $a>0$ such that $f(a)>0$ and let $v(r)$ be the solution to (17) subject to initial conditions $v(0)=a$ and $v^{\prime}(0)=0$. Integrating identity $(17)$ over $[0 ; r], r<R$, we obtain

$$
A\left(\left|v^{\prime}(r)\right|\right) v^{\prime}(r)=\frac{1}{q^{n-1}(r)} \int_{0}^{r} q^{n-1}(s) f(v(s)) d s, \quad 0<r<R .
$$

Then $v^{\prime}(r) \geq 0$ for $0 \leqslant r<R$, since $A\left(v^{\prime}(r)\right) v^{\prime}(r) \geq 0$. Let us show that the solution $v(r)$ exists on $[0 ; \infty)$. Suppose the opposite, namely, that the solution $v(r)$ is defined on a finite segment $[0 ; R), R<\infty$.

Since $v^{\prime}(r) \geq 0$ as $0 \leqslant r<R$, then $v(R-0)$ has the values in the segment $(0 ; \infty]$. Consider the case $v(R-0)<\infty$. Then it follows from (18) that $v^{\prime}(R-0)<\infty$ and therefore the solution $v$ can be extended to the right through $R$ (see [9], pp. 58-62). It contradicts to the choice of $R$. Hence, $v(R-0)=\infty$.

Let us represent (17) as

$$
\left(A\left(\left|v^{\prime}(r)\right|\right) v^{\prime}(r)\right)^{\prime}+(n-1) \frac{q^{\prime}(r)}{q(r)} A\left(\left|v^{\prime}(r)\right|\right) v^{\prime}(r)=f(v(r)) .
$$

Since $v^{\prime}(r) \geq 0$ as $0 \leqslant r<R$ and $q^{\prime}(r) \geq 0$, then

$$
\left(A\left(\left|v^{\prime}(r)\right|\right) v^{\prime}(r)\right)^{\prime} \leqslant f(v(r)) .
$$

Multiplying the latter inequality by $v^{\prime}(r)$ and integrating it over the segment $[0 ; r], r<R$, we obtain

$$
\Psi\left(v^{\prime}(r)\right) \leqslant \int_{v(0)}^{v(r)} f(s) d s
$$

It yields

$$
\left(\Phi\left(\int_{v(0)}^{v(r)} f(s) d s\right)\right)^{-1} v^{\prime}(r) \leqslant 1, \quad r>0
$$


Integrating over the segment $[0 ; r]$ as $r<R$ once again, we have

$$
\int_{v(0)}^{v(r)}\left(\Phi\left(\int_{v(0)}^{s} f(t) d t\right)\right)^{-1} d s \leqslant r, \quad r>0 .
$$

Letting $r \rightarrow R$, we obtain

$$
\int_{v(0)}^{\infty}\left(\Phi\left(\int_{v(0)}^{s} f(t) d t\right)\right)^{-1} d s \leqslant R<\infty
$$

that contradicts (7). Therefore, the solution $v$ to equation (17) subject to initial conditions $v(0)=a$ and $v^{\prime}(0)=0$ there exists on the ray $[0, \infty)$. By the arbitrariness of $a>0$ we obtain the continuum of various positive solutions to equation (17) and thus to inequality (1). The proof is complete.

\section{BIBLIOGRAPHY}

1. A. Grigor'yan. Analytic and geometric background of recurrence and non-explosion of the Brownian motion on Riemannian manifolds. // Bull. Amer. Math. Soc. 1999. V. 36, No. 2. P. 135-249.

2. A.G. Losev. Certain Liouville theorems for Riemannian manifolds of a special form. // Izv. VUZov. Matem. 1991. No. 12. P. 15-24. [Soviet Math. 1991. V. 35, No. 12. P. 15-23.]

3. A.G. Losev, E.A. Mazepa. On the asymptotic behavior of solutions of some elliptic-type equations on noncompact Riemannian manifolds . // Izv. VUZov. Matem. 1999. No. 6. P. 41-49. [Russ. Math. 1999. V. 43, No. 6. P. 39-47.]

4. A.G. Losev, Yu.S. Fedorenko Positive solutions of quasilinear elliptic inequalities on noncompact Riemannian manifolds. // Matem. zametki. 2007. V. 81, No. 6. P. 867-878. [Math. notes. 2007. V. 81, No. 6. P. 778-787.]

5. Y. Naito, H. Usami. Entire solutions of the inequality $\operatorname{div}(A(|D u|) D u) \geq f(u) / /$ Math. Z. 1997. V. 255, No. 1. P. 167-175.

6. Keller J.B. On solutions of $\Delta u=f(u) / /$ Commun. Pure Appl. Math. 1957. V 10, No. 4. P. 503-510.

7. R. Osserman On the inequality $\Delta u \geq f(u)$. // Pac. J. Math. 1957. V. 7, No. 4. P. 1641-1647.

8. T. Kusano, C.A. Swanson. Radial entire solutions of a class of quasilinear elliptic equations // J. Diff. Eqs. 1990. V. 83, No. 2. P. 379-399.

9. Yu.N. Bibikov. The course of ordinary differential equations. Vysshaya shkola, Moscow. 1991. (in Russian).

Alexnader Georgievich Losev,

Volgograd State University,

Universtitetskii av., 100

400062, Volgograd, Russia

E-mail: alexander.losev@volsu.ru

Elena Alexeevna Mazepa,

Volgograd State University,

Universtitetskii av., 100

400062, Volgograd, Russia

E-mail: lmazepa@rambler.ru 